\title{
Heavy quarks in MRST/MSTW global fits
}

\author{
R. S. Thorne ${ }^{\mathrm{a}}$

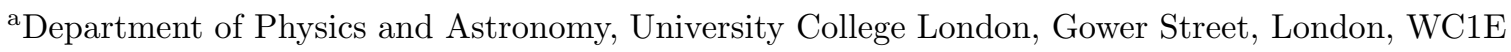 \\ 6BT, United Kingdom
}

I discuss the procedure of interpolating from scales lower than, or similar to the quark masses $m_{H}$, to those much higher than $m_{H}$ via the use of a General Mass Variable flavour Number Scheme (GM-VFNS). The precise definitions used in MRST and MSTW global fits are explained and the consequences for the extracted parton distributions investigated.

\section{Introduction}

The correct treatment of heavy flavours in an analysis of parton distributions is essential for precision measurements at hadron colliders. Cross sections at colliders depend on precise knowledge of the heavy quark parton densities, but a reliable determination of the heavy-flavour distributions is also important because of the "knock-on" effect on the other parton densities. It has become clear in recent years that it is a delicate issue to obtain a proper treatment of heavy flavours. There are various choices which can be made, and also many ways in which subtle mistakes can occur. Both the choices and the mistakes can lead to changes in parton distributions which may be similar to, or even greater than, the quoted uncertainties - though the mistakes usually lead to the more dramatic changes. Hence, I will here provide a full description of our procedure, along with a comparison to alternatives and some illustrations of pitfalls which must be avoided. I begin by describing the two distinct regimes for heavy quarks where the pictures are relatively simple. These are the so-called Fixed Flavour Number Scheme (FFNS) and zero-mass Variable Flavour Number Scheme (ZM-VFNS).

\subsection{FFNS and ZM-VFNS}

First, there is the region where the hard scale of the process is similar to, or smaller than, the quark mass ${ }^{1}$, i.e. $Q^{2} \lesssim m_{H}^{2}$. In this case it is most

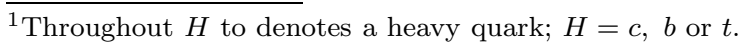

natural to describe the massive quarks as final state particles, and not as partons within the proton. This requirement defines the FFNS, where only light quarks are partons, and the number of flavours is fixed. We label the number of quark flavours appearing as parton distributions by $n_{f}$. Note, however, that there are FFNS with different numbers of active flavours $n_{f}$. The number $n_{f}$ is normally equal to 3 , where up, down and strange are the light quarks, but we can treat charm as a light quark while bottom remains heavy at high scales, i.e. $n_{f}=4$, and it may also be equal to 5 . In each example of an FFNS the structure functions are given by ${ }^{2}$

$F_{i}\left(x, Q^{2}\right)=C_{i, k}^{\mathrm{FF}, n_{f}}\left(\frac{Q^{2}}{m_{H}^{2}}\right) \otimes f_{i, k}^{n_{f}}\left(Q^{2}\right)$.

This approach contains all the $m_{H}$-dependent contributions, and is conceptually simple so is frequently used in analyses of structure functions. Even in this case one must be careful to be selfconsistent in defining all quantities, i.e. parton distributions, coefficient functions and coupling constant in the same renormalization schemes. The mistake made by not doing so can lead to errors in the gluon distribution similar to the uncertainty [1].

\footnotetext{
${ }^{2}$ I use the convention that the factorization scale and renormalization scale are both equal to $Q^{2}$. It is also the choice made in the analysis. Alternative choices are possible, and cause no problems in principle, but can lead to considerable technical complication.
} 
Despite its conceptual simplicity, the FFNS has problems. It does not sum $\alpha_{S}^{m} \ln ^{l} Q^{2} / m_{H}^{2}, l \leq m$ terms in the perturbative expansion, and the accuracy of the fixed-order expansion becomes increasingly uncertain as $Q^{2}$ increases above $m_{H}^{2}$. There are additional practical issues. Since calculations including full mass dependence are complicated, there are only a few cross sections known even to NLO in $\alpha_{S}$ within this framework, so the resulting parton distributions are not universally useful. Even for neutral-current structure functions, the FFNS coefficient functions are known only up to NLO [2], and are not calculated at $\mathrm{NNLO}$ - i.e. the $\alpha_{S}^{3}$ coefficient $^{3}, C_{2, H g}^{\mathrm{FF}, n_{f}, 3}$, for $F_{2}$ is unknown, so one cannot determine parton distributions at NNLO in this scheme.

The problems of the FFNS are solved in the so-called zero-mass Variable Flavour Number Scheme (ZM-VFNS). Here, the heavy quarks evolve according to the splitting functions for massless quarks and the resummation of the large logarithms in $Q^{2} / m_{H}^{2}$ is achieved by the introduction of heavy-flavour parton distributions and the solution of the evolution equations. It assumes that at high scales, $Q^{2} \gg m_{H}^{2}$, the massive quarks behave like massless partons, and the coefficient functions are simply those in the massless limit, e.g. for structure functions

$F_{i}\left(x, Q^{2}\right)=C_{i, j}^{\mathrm{ZMVF}, n_{f}} \otimes f_{j}^{n_{f}}\left(Q^{2}\right)$,

where $n_{f}-3$ is the number of active heavy quarks, with masses above some transition point for turning on the heavy flavour distribution, typically at a scale similar to $m_{H}^{2}$. This is technically simpler than the FFNS, and many more cross sections are known in this scheme. The nomenclature of "zero-mass" is a little misleading because some mass dependence is included. The parton densities in different quark number regimes are related to each other perturbatively, i.e.

$f_{j}^{n+1}\left(Q^{2}\right)=A_{j k}\left(\frac{Q^{2}}{m_{H}^{2}}\right) \otimes f_{k}^{n}\left(Q^{2}\right)$,

where the matrix elements $A_{j k}\left(Q^{2} / m_{H}^{2}\right)$ [3] containing $\ln \left(Q^{2} / m_{H}^{2}\right)$ terms are known to NNLO,

\footnotetext{
${ }^{3} \mathrm{I}$ add a subscript $H$ to distinguish the $g \rightarrow H$ coefficient function $C_{H g}$ from the usual coefficient function $C_{g}$ describing the $g \rightarrow q$ transition of light quarks.
}

i.e. $\mathcal{O}\left(\alpha_{S}^{2}\right)$. They relate $f_{i}^{n}\left(Q^{2}\right)$ and $f_{i}^{n+1}\left(Q^{2}\right)$, guaranteeing the correct evolution for both regimes. At $\mathrm{NLO}$ in the $\overline{\mathrm{MS}}$ scheme they constrain the heavy quarks to evolve from a zero value at $Q^{2}=m_{H}^{2}$, and the other partons to be continuous at this choice of transition point, hence making it the natural choice.

The ZM-VFNS has many advantages. However, it has the failing that it simply ignores $\mathcal{O}\left(m_{H}^{2} / Q^{2}\right)$ corrections to the coefficient functions, and hence is inaccurate in the region where $Q^{2}$ is not so much greater than $m_{H}^{2}$. The nomenclature scheme is misleading since it usually implies an alternative choice in ordering the expansion, or a particular separation of contributions between coefficient functions and parton densities, i.e. the inherent ambiguity in a perturbative QCD calculation allows a choice, the effects of which become increasing smaller as higher orders are included. The ZM-VFNS involves missing out $\mathcal{O}\left(m_{H}^{2} / Q^{2}\right)$ contributions completely, and there is a permanent error of this order. The error induced by fitting to HERA structure function data using a ZM-VFNS was shown to be up to $\sim 6 \%$ in the small- $x$ light-quark distributions by CTEQ in [4], resulting in a systematic error of $\sim 8 \%$ in predictions for vector boson production at the LHC. This NLO result seems to provide ample evidence for the use of a general mass Variable Flavour Number Scheme (GM-VFNS) to provide default parton distributions, and CTEQ have since adopted this convention. However, the inherent problems with the ZM-VFNS are thrown into particularly sharp relief at NNLO. At $\mathcal{O}\left(\alpha_{S}^{2}\right)$ the $A_{j k}\left(Q^{2} / m_{H}^{2}\right)$ are no longer zero at $Q^{2}=m_{H}^{2}$, and lead to discontinuities in the parton densities. This is similar to the discontinuity at $\mu^{2}=m_{H}^{2}$ in $\alpha_{S}\left(\mu^{2}\right)$ at NNLO. For the coupling constant this discontinuity is rather small. The corresponding discontinuities can be significant for the gluon, about $10 \%$, but it turns out that $c\left(x, m_{c}^{2}\right)$ is considerably negative at small $x$, see e.g. Fig. 3 of [5]. The effect of the NNLO massless coefficient function is to make this worse rather than better at $x \sim 0.0001-0.01$, and the charm contribution to $F_{2}\left(x, Q^{2}\right)$ is negative at the transition point to such a degree that there is an $\mathcal{O}(10 \%)$ discontinuity in the total structure function at $Q^{2}=m_{c}^{2}$, 
as illustrated in Fig. 1.

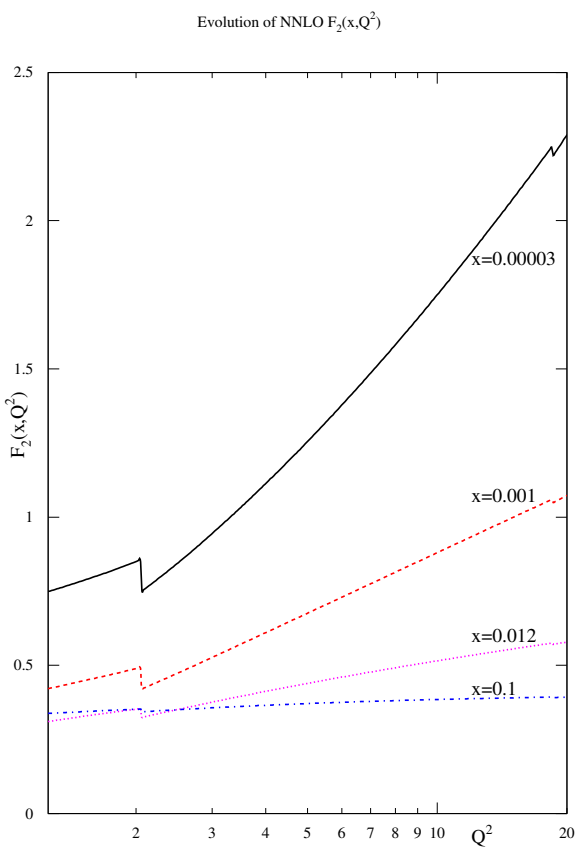

Figure 1. The discontinuity in $F_{2}\left(x, Q^{2}\right)$ using the ZM-VFNS at NNLO.

Hence, for a precise analysis of structure function, and other data, one must use a General Mass Variable Flavour Number Scheme which smoothly connects the two well-defined limits of $Q^{2} \leq m_{H}^{2}$ and $Q^{2} \gg m_{H}^{2}$. A definition of a GM-VFNS was first proposed in [6] - the ACOT scheme, and the MRST group have been using the alternative TR scheme [7] as the default since MRST98[8]. However, despite the above reasoning, a GM-VFNS is not always used as default even today. This is partially due to the extra complication compared to the simple ZM-VFNS. However, part of the reason may be because the definition is not unique. The reasons for this and the consequences will now be discussed, along with a detailed outline of the prescription used in the MRST/MSTW analysis. A less detailed, but more introductory and comparative discussion of the schemes used by MRST/MSTW and CTEQ can be found in [9].

\section{General-Mass Variable Flavour Number Schemes}

A GM-VFNS is defined by demanding equivalence of the $n$ (FFNS) and $n+1$-flavour (GMVFNS) descriptions above the transition point for the new parton distribution (they are by definition identical below this), at all orders, i.e.

$$
\begin{aligned}
& F_{i}\left(x, Q^{2}\right)=C_{i, k}^{\mathrm{FF}, n}\left(\frac{Q^{2}}{m_{H}^{2}}\right) \otimes f_{k}^{n}\left(Q^{2}\right) \\
&=C_{i, j}^{\mathrm{VF}, n+1}\left(\frac{Q^{2}}{m_{H}^{2}}\right) \otimes f_{j}^{n+1}\left(Q^{2}\right) \\
& \equiv C_{i, j}^{\mathrm{VF}, n+1}\left(\frac{Q^{2}}{m_{H}^{2}}\right) \otimes A_{j k}\left(\frac{Q^{2}}{m_{H}^{2}}\right) \otimes f_{k}^{n}\left(Q^{2}\right) .
\end{aligned}
$$

The description where the number of active partons is taken to be $n_{f}=n$ must be identical to that when it increases, i.e. $n_{f}=n+1$. Hence, the GM-VFNS coefficient functions satisfy ${ }^{4}$

$C_{i, k}^{\mathrm{FF}, n}\left(\frac{Q^{2}}{m_{H}^{2}}\right)=C_{i, j}^{\mathrm{VF}, n+1}\left(\frac{Q^{2}}{m_{H}^{2}}\right) \otimes A_{j k}\left(\frac{Q^{2}}{m_{H}^{2}}\right)$,

which, for example, at $\mathcal{O}\left(\alpha_{S}\right)$ gives for $F_{2}\left(x, Q^{2}\right)$

$$
\begin{aligned}
C_{2, g}^{\mathrm{FF}, n, 1}\left(\frac{Q^{2}}{m_{H}^{2}}\right)= & C_{2, H H}^{\mathrm{VF}, n+1,0}\left(\frac{Q^{2}}{m_{H}^{2}}\right) \otimes P_{q g}^{0} \ln \left(\frac{Q^{2}}{m_{H}^{2}}\right)(6) \\
& +C_{2, g}^{\mathrm{VF}, n+1,1}\left(\frac{Q^{2}}{m_{H}^{2}}\right) .
\end{aligned}
$$

The GM-VFNS coefficient functions, $C^{\mathrm{VF}}$, are constrained to tend to the massless limits as $Q^{2} / m_{H}^{2} \rightarrow \infty$ and the $A_{j k}\left(Q^{2} / m_{H}^{2}\right)$ are such that this happens self consistently. However, the $C_{i, j}^{\mathrm{VF}, n_{f}}\left(Q^{2} / m_{H}^{2}\right)$ are only uniquely defined in this massless limit. For finite $Q^{2} / m_{H}^{2}$ one can swap $\mathcal{O}\left(m_{H}^{2} / Q^{2}\right)$ terms between $C_{2, H H}^{\mathrm{VF}, n+1,0}\left(Q^{2} / m_{H}^{2}\right)$ and $C_{2, g}^{\mathrm{VF}, n+1,1}\left(Q^{2} / m_{H}^{2}\right)$ while maintaining the exact definition in (7). This general feature applies to all relationships in (5). Although the equivalence (5) was first pointed out in general

\footnotetext{
${ }^{4}$ It is implicit that the coupling constant is a function of $n$ flavours on the left-hand side and of $n+1$ flavours on the right-hand side.
} 
in [3], and (7) is effectively used in defining the original ACOT scheme, the freedom to swap $\mathcal{O}\left(m_{H}^{2} / Q^{2}\right)$ terms without violating the definition of a GM-VFNS was first noticed in [7] and put to use to define the TR scheme, as described below. This freedom to redistribute $\mathcal{O}\left(m_{H}^{2} / Q^{2}\right)$ terms can be classified as a change in scheme since it leads to an ambiguity in the result at a fixed order, but the ambiguity becomes higher order if the order of the calculation increases, much like the renormalization scheme (and scale) ambiguity. Moreover, it is a change of scheme which does not change the definition of the parton distributions, only the coefficient functions. This is perhaps a surprising result, which occurs because there is a redundancy in (5), there being one more coefficient function above the transition point than below, i.e. that for the heavy quark.

The original ACOT prescription calculated the coefficient functions for single heavy quark scattering from a virtual photon exactly. This might seem the most natural definition. However, it assumes that immediately above the transition point a single heavy quark or antiquark can exist in isolation. Each coefficient function violates the real physical threshold $W^{2}>4 m_{H}^{2}$, since only one heavy quark is produced in the final state. Moreover, this definition requires the calculation of mass-dependent coefficient functions which becomes progressively more difficult at higher orders. In the TR scheme [7] $C_{2, H H}^{\mathrm{VF}, n_{f}, 0}\left(Q^{2} / m_{H}^{2}\right)$ was defined by the constraint that $\left(d F_{2}^{H} / d \ln Q^{2}\right)$ was continuous at the transition point (in the gluon sector). This imposed the correct threshold dependence on all coefficient functions and improved the smoothness at $Q^{2}=m_{H}^{2}$, and did not involve the calculation of new mass-dependent diagrams. However, it required the convolution of the formal inverse of splitting functions with coefficient functions, which itself becomes technically difficult at higher orders.

Since these early definitions there have been various modifications, including a precise definition of an ACOT-like scheme up to NNLO by Chuvakin, Smith and van Neerven $[10]^{5}$. A major

\footnotetext{
${ }^{5}$ Indeed in [10] it is pointed out that at NNLO a further complication appears for the first time. There are
}

simplification was achieved when the flexibility in the choice of heavy-quark coefficient functions was used to define the $\operatorname{ACOT}(\chi)$ prescription [11], which in the language used in this paper (and in [7]) would be defined by

$C_{2, H H}^{\mathrm{VF}, n_{f}, 0}\left(z, \frac{Q^{2}}{m_{H}^{2}}\right)=z\left(1+4 \frac{m_{H}^{2}}{Q^{2}}\right) \delta\left(z-\frac{Q^{2}}{Q^{2}+4 m_{H}^{2}}\right)$

This gives the LO definition

$F_{2}^{H, 0}\left(x, Q^{2}\right)=(h+\bar{h})\left(x / x_{\max }, Q^{2}\right)$,

where $x_{\max }=Q^{2} /\left(Q^{2}+4 m_{H}^{2}\right)$. It automatically reduces to the massless limit $C_{2, H H}^{\mathrm{ZM}, n_{f}, 0}(z)=\delta(1-$ $z$ ) for $Q^{2} / m_{H}^{2} \rightarrow \infty$, and also imposes the true physical threshold

$W^{2}=Q^{2}(1-x) / x \geq 4 m_{H}^{2}$.

This choice of the LO heavy-flavour coefficient function has been adopted in our current prescription, the $\mathrm{TR}^{\prime}$ scheme, described in detail in [12]. For the GM-VFNS to remain simple (and physical) at all orders, $m$, it is necessary to choose

$C_{2, H H}^{\mathrm{VF}, n_{f}, m}\left(Q^{2} / m_{H}^{2}, z\right)=C_{2, H H}^{\mathrm{ZM}, n_{f}, m}\left(z / x_{\max }\right)$,

which is the implicit $\operatorname{ACOT}(\chi)$ definition, and is our choice. It removes one of the sources of ambiguity in defining a GM-VFNS. However, there are others.

One major issue in a complete definition of the GM-VFNS is that of the ordering of the perturbative expansion. This ambiguity comes about because the ordering in $\alpha_{S}$ for $F_{2}^{H}\left(x, Q^{2}\right)$ is different for the number of active flavours $n_{f}=n$ and $n_{f}=n+1$ regions.

$$
\begin{array}{cc}
n \text { - flavour } & n+1 \text { - flavour } \\
\text { LO } \quad \frac{\alpha_{S}}{4 \pi} C_{2, H g}^{\mathrm{FF}, n, 1} \otimes g^{n} & C_{2, H H}^{\mathrm{VF}, n+1,0} \otimes(h+\bar{h})
\end{array}
$$

$\ln ^{3}\left(Q^{2} / m_{H}^{2}\right)$ divergences at $\mathcal{O}\left(\alpha_{S}^{2}\right)$ coming from gluon splitting into heavy quark-antiquark pairs. These divergences arise from heavy quark emission diagrams which cancel with opposite ones originating from virtual heavy quarks loops in the "light quark" coefficient functions. This cancellation is achieved in a physically meaningful manner by imposing a cut on the softness of the heavy quark final state in the former process. In practice, after cancellation both contributions are very small so we currently include the total in the "light quark" sector. In the $\mathcal{O}\left(\alpha_{S}^{2}\right)$ FFNS they are usually combined instead in the "heavy quark" contribution. 
with generalization to higher orders. Switching directly from $n$ flavours at fixed order to $n+1$ at fixed order leads to a discontinuity in $F_{2}^{H}\left(x, Q^{2}\right)$. As with the discontinuities in the ZM-VFNS this is not just a problem in principle - the discontinuity is comparable to the errors on data.

Hence, any definition of a GM-VFNS must make some decision how to deal with this, and the ACOT-type schemes have always made a different choice to that for the TR-type schemes used in our analyses. The ACOT-type schemes simply define the same order of $\alpha_{S}$ both below and above the transition point. For example at NLO the definition is

$$
\begin{aligned}
& F_{2}^{H}\left(x, Q^{2}\right)=\frac{\alpha_{S}}{4 \pi} C_{2, H g}^{\mathrm{FF}, n, 1} \otimes g^{n} \\
& \rightarrow \frac{\alpha_{S}}{4 \pi}\left(C_{2, H H}^{\mathrm{VF}, n+1,1} \otimes(h+\bar{h})+C_{2, H g}^{\mathrm{FF}, n+1,1} \otimes g^{n+1}\right) .
\end{aligned}
$$

This clearly maintains continuity in the structure function across the transition point. However, it only contains information on LO heavy flavour evolution below $Q^{2}=m_{H}^{2}$, since $C_{2, H g}^{\mathrm{FF}, n, 1}$ only contains information on the LO splitting function, but the heavy quarks evolve using NLO splitting functions above $Q^{2}=m_{H}^{2}$ - a big change at small $x$. The TR scheme, and all subsequent variations used in our analyses, try to maintain the correct ordering in each region as closely as possible. For example at LO

$$
\begin{aligned}
F_{2}^{H}\left(x, Q^{2}\right)= & \frac{\alpha_{S}\left(Q^{2}\right)}{4 \pi} C_{2, H g}^{\mathrm{FF}, n, 1}\left(\frac{Q^{2}}{m_{H}^{2}}\right) \otimes g^{n}\left(Q^{2}\right)(13) \\
& \rightarrow \quad \frac{\alpha_{S}\left(m_{H}^{2}\right)}{4 \pi} C_{2, H g}^{\mathrm{FF}, n, 1}(1) \otimes g^{n}\left(m_{H}^{2}\right) \\
& +\quad C_{2, H H}^{\mathrm{VF}, n+1,0}\left(\frac{Q^{2}}{m_{H}^{2}}\right) \otimes(h+\bar{h})\left(Q^{2}\right),
\end{aligned}
$$

i.e. we freeze the $\mathcal{O}\left(\alpha_{S}\right)$ term when going upwards through $Q^{2}=m_{H}^{2}$. This generalises to higher orders by freezing the term with the highest power of $\alpha_{S}$ in the definition for $Q^{2}<m_{H}^{2}$ when moving upwards above $m_{H}^{2}$. Hence, the definition of the ordering is consistent within each region, except for the addition of a constant term (which does not affect evolution) above $Q^{2}=m_{H}^{2}$ which becomes progressively less important at higher $Q^{2}$, and whose power of $\alpha_{S}$ increases as the order of the perturbative expansion increases.
This definition means that in order to define our VFNS at NNLO [12] we need to use the $\mathcal{O}\left(\alpha_{S}^{3}\right)$ heavy-flavour coefficient functions for $Q^{2} \leq m_{H}^{2}$ (and that the contribution will be frozen for $\left.Q^{2}>m_{H}^{2}\right)$. As mentioned above, these coefficient functions are not yet calculated. However, we can model this contribution using the known leading threshold logarithms [13] and leading $\ln (1 / x)$ terms derived from the $k_{T}$-dependent impact factors [14]. This results in a significant contribution at small $Q^{2}$ and $x$ with some model dependence, though variation in the free parameters does not lead to a large change. ${ }^{6}$ Up to this small model-dependence we have a full NNLO GM-VFNS with automatic continuity of structure functions across heavy flavour transition points. ${ }^{7}$ This is certainly the most complete treatment of heavy-flavour effects currently used in any NNLO analysis.

\section{Scheme dependence}

Although all definitions of the GM-VFNS become very similar at very high $Q^{2}$, the difference in choice can be phenomenologically important. For example, our definition is effectively including exactly one higher order than ACOT-type schemes for $Q^{2}<m_{H}^{2}$, and the value of this contribution at $Q^{2}=m_{H}^{2}$ is carried to higher $Q^{2}$. Since at small $x$ the higher orders in $\alpha_{S}$ are accompanied by large corrections this leads to large differences below the transition point, which are still important a little way above the transition point. This is shown for $F_{2}^{c}\left(x, Q^{2}\right)$ in Fig. 2 of [12] where the two choices are shown for the same parton distributions. More clearly, this difference in the definition of the ordering is the main difference in the NLO predictions from MRST and

\footnotetext{
${ }^{6}$ It should be stressed that this model is only valid for the region $Q^{2} \leq m_{H}^{2}$, and contains no information on the large $Q^{2} / m_{H}^{2}$ limits of the coefficient functions.

${ }^{7}$ There are $\mathcal{O}\left(\alpha_{S}^{3}\right)$ discontinuities due to terms such as $C_{2, H H}^{\mathrm{VF}, n+1,1} \otimes(h+\bar{h})$ and $C_{2, H g}^{\mathrm{VF}, n+1,1} \otimes g^{n+1}$, i.e. $\mathcal{O}\left(\alpha_{S}\right)$ coefficient functions convoluted with $\mathcal{O}\left(\alpha_{S}^{2}\right)$ discontinuities in partons. These would be cancelled at NNNLO by discontinuities in $\mathcal{O}\left(\alpha_{S}^{3}\right)$ coefficient functions. In practice the imposition of the correct threshold behaviour in all coefficient functions minimises these effects and they are very small.
} 
CTEQ in the comparison to H1 data on $F_{2}^{b}\left(x, Q^{2}\right)$ [15], shown in Fig. 2.

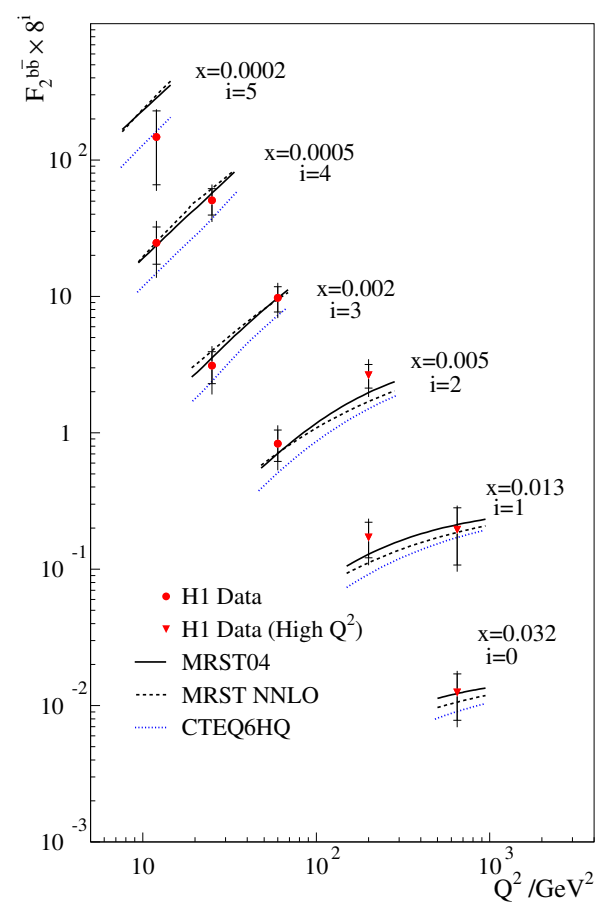

Figure 2. Comparison of the MRST and CTEQ predictions for $F_{b}^{c}\left(x, Q^{2}\right)$ compared with published H1 data [15].

The inclusion of the complete GM-VFNS in a global fit at NNLO first appeared in [5], and led to some important changes compared to the previous NNLO analyses, which had a much more approximate inclusion of heavy flavours (explained in the Appendix of [16]). A consequence of including the positive $\mathcal{O}\left(\alpha_{S}^{3}\right)$ coefficient functions at low $Q^{2}$ is that the NNLO $F_{2}^{c}\left(x, Q^{2}\right)$ automatically starts from a higher value at low $Q^{2}$. However, at high $Q^{2}$, the structure function is dominated by $(c+\bar{c})\left(x, Q^{2}\right)$. This has started evolving from a significantly negative value at $Q^{2}=m_{c}^{2}$. The partons in an NNLO fit readjust so that the light flavours evolve similarly to those at NLO, in order to fit the data. Since the heavy flavour quarks evolve at the same rate as light quarks, but at NNLO start from a negative starting value, they remain lower than at NLO for higher $Q^{2}$. Hence, there is a general trend $-F_{2}^{c}\left(x, Q^{2}\right)$ is flatter in $Q^{2}$ at NNLO than at NLO, as shown in Fig. 4 of [5]. It is also flatter than our previous approximate NNLO prescription. This had an important effect on the gluon distribution. As seen in Fig. 5 of [5], it led to a larger gluon for $x \sim 0.0001-0.01$, as well as a larger value of $\alpha_{S}\left(M_{Z}^{2}\right)$, both compensating for the naturally flatter evolution, and consequently leading to more evolution of the light quark sea. Both the gluon and the light quark sea were up to $6-7 \%$ greater than in the 2004 set [17] for $Q^{2}=10^{4} \mathrm{GeV}^{2}$, the increase maximising at $x=0.0001-0.001$. As a result there was a $6 \%$ increase in the predictions for $\sigma_{W}$ and $\sigma_{Z}$ at the LHC. This surprisingly large change is a correction rather than a reflection of the uncertainty due to the freedom in choosing heavy flavour schemes.

The 2006 MRST NNLO parton update [5] was made because this was the first time the heavy flavour prescription had been treated precisely at NNLO and also because there was previously no MRST NNLO set with uncertainties. The data used in the analysis were very similar to the 2004 set, and since a consistent GM-VFNS was already used at NLO, and a set with uncertainties already existed, no new corresponding release of an NLO set was made along with the 2006 NNLO set. With the benefit of hindsight, it would be interesting to check the effect on the distributions due to the change in the prescription for the GM-VFNS at NLO without complicating the issue by also changing many other things in the analysis. To this end we have obtained an unofficial "MRST2006 NLO" set, which is fit to exactly the same data as the MRST2006 NNLO set.

The comparison of the up quark and gluon distributions for the "MRST2006 NLO" set and the MRST2004 NLO set, i.e. the comparable plot to Fig. 5 of [5] for NNLO, is shown in Fig. 3. As can be seen it leads to the same trend for the partons as at NNLO, i.e. an increase in the small- $x$ gluon and light quarks, but the effect is much 

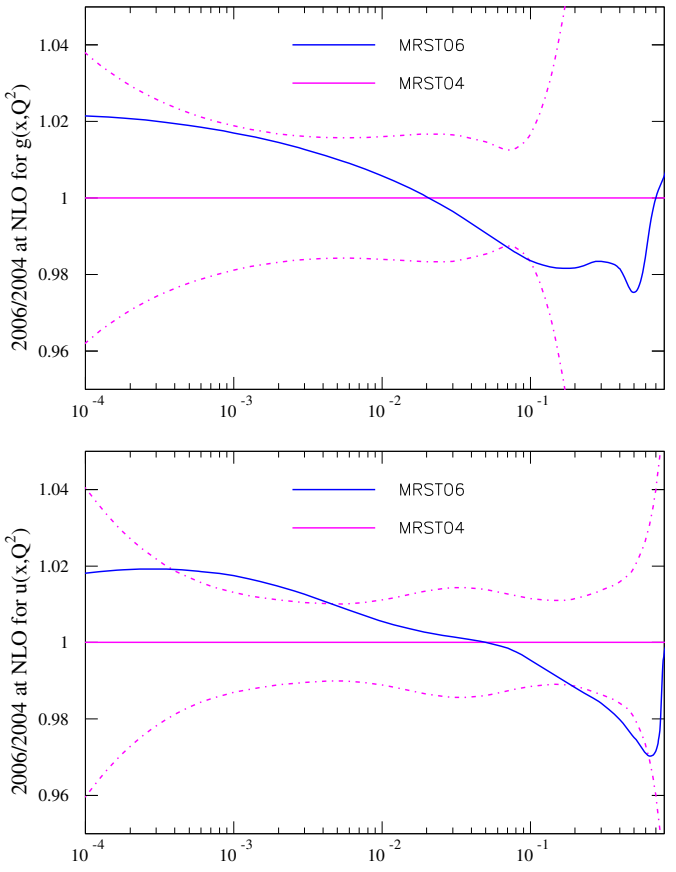

Figure 3. A comparison of the unpublished "MRST2006 NLO" parton distributions to the MRST2004 NLO distributions at $Q^{2}=10^{4} \mathrm{GeV}^{2}$. In order to illustrate the significance of the size of the differences, the uncertainty on the MRST2001 distributions is used for the 2004 distributions. The corresponding comparison at NNLO can be seen in Fig. 5 of [5].

smaller - a maximum of a $2 \%$ change. Also, the value of the coupling constant increases by 0.001 from the 2004 value of $\alpha_{S}\left(M_{Z}^{2}\right)=0.120$. Again, this is similar to, but smaller than, the change at NNLO. Hence, we can conclude that the change in our choice of the heavy-flavour coefficient function alone leads to changes in the distributions of up to $2 \%$, and since the change is simply a freedom we have in making a definition, this is a theoretical uncertainty on the partons, much like the frequently invoked scale uncertainty. Like the latter, it should decrease as we go to higher orders. The ambiguity simultaneously moves to higher order, but it is difficult to check this explicitly since our main reason for making our change in the choice of heavy-quark coefficient functions was the difficulty of applying the original procedure in [7] at NNLO. Certainly an absolute maximum of $2 \%$ of the $6-7 \%$ change, in the predictions for $\sigma_{W}$ and $\sigma_{Z}$ at the LHC in going from the 2004 to the 2006 NNLO parton sets, is due to true ambiguities and the remaining $5 \%$ is due to the correction of the flaws in the previous approach. The "MRST2006 NLO" distributions lead to a $2 \%$ increase in the predictions for $\sigma_{W}$ and $\sigma_{Z}$ at the LHC compared to MRST2004 NLO, but there is very little change at the Tevatron, where the typical values of $x$ probed are nearly an order of magnitude higher.

\section{Longitudinal Structure Function}

One also has to be careful in defining the GMVFNS for the longitudinal structure function. In one sense this is not so important since the longitudinal contribution to the total cross section measured in structure function experiments is small, and the errors on direct measurements are comparatively large. However, the importance is increased by the larger ambiguity inherent in the definition of $F_{L}$ as compared to $F_{2}$.

This large ambiguity occurs because, if one calculates the coefficient function for a single massive quark scattering off a virtual photon, there is an explicit zeroth-order contribution

$C_{L, H H}^{\mathrm{VF}, n_{f}, 0}\left(z, Q^{2} / m_{H}^{2}\right)=z \frac{4 m_{H}^{2}}{Q^{2}} \delta\left(z-\frac{Q^{2}}{Q^{2}+m_{H}^{2}}\right)$.

This disappears at high $Q^{2}$ and the correct zeromass limit is reached.

Such a zeroth-order coefficient function is used in the original ACOT definition of a GM-VFNS, and leads to a peculiar behaviour of $F_{L}^{c}$ just above $Q^{2}=m_{c}^{2}$. It is convoluted with the heavy flavour distribution, which for $Q^{2}$ just above $m_{c}^{2}$ is small in magnitude. However, the coefficient function is large near $m_{c}^{2}$, while the unsubtracted (i.e. FFNS) gluon and singlet-quark coefficient functions are suppressed by a factor of $v^{3}$, where $v$ is the velocity of the heavy quark in the centre-of- 
mass frame, and are very small for low $Q^{2}$. This means that this zeroth-order heavy-flavour contribution dominates just above $Q^{2}=m_{c}^{2}$, despite the fact that if a $c \bar{c}$ pair has to be created, as it must in reality, the contribution is absent.

The contribution from $C_{L, c c}^{\mathrm{VF}, n_{f}, 0} \otimes(c+\bar{c})$ turns on rapidly just above $m_{c}^{2}$, dominating other contributions, then dies away as $m_{H}^{2} / Q^{2}$ becomes small. This leads to a distinct bump in $F_{L}^{c}\left(x, Q^{2}\right)$ for $Q^{2}$ just above $m_{c}^{2}$, as pointed out in [7]. In principle this cancels between orders in a properly defined GM-VFNS, as this contribution implicitly appears in the subtraction terms for the gluon and singlet-quark coefficient functions with opposite sign to its explicit contribution. However, the cancellation is imperfect at finite order, and even the partially cancelled contribution dominates at NLO. At NNLO, where heavy-flavour distributions begin at $Q^{2}=m_{c}^{2}$ with negative values, the "bump" in $F_{L}^{c}\left(x, Q^{2}\right)$ is negative, as illustrated in Fig. 18 of [10], highlighting the unphysical nature of this contribution.

Hence, as in [7] we choose to ignore the explicit single heavy quark-photon scattering results. We define the longitudinal sector in what seems to us to be the most physical generalization of the definition for $F_{2}^{H}\left(x, Q^{2}\right)$, as explained in [12]. The heavy-quark coefficient functions are simply those for the light quarks, with the upper limit of integration moved from 1 to $x_{\max }=Q^{2} /\left(Q^{2}+4 m_{H}^{2}\right)$. Thus the physical threshold of $W^{2} \geq 4 m_{H}^{2}$ is contained in all terms, and there are no spurious zeroth-order terms. These could only make a contribution if one works in the framework of single heavy quark scattering in the region of low $Q^{2}$ where the parton model for the heavy quark is least appropriate. The definition of the SACOT type scheme [18], and particularly the $\operatorname{SACOT}(\chi)$ scheme used in the global fits [4] also avoids this undesirable zeroth-order coefficient function.

\section{Charged Currents}

The general procedure for the GM-VFNS for charged-current deep inelastic scattering works on the same principles as for neutral currents - one can now produce a single charm quark from a strange quark so the threshold is now at $x_{\max }=1 /\left(1+m_{c}^{2} / Q^{2}\right)$. However, as explained in [12], there is a complication because the massive FFNS coefficient functions are not known at $\mathcal{O}\left(\alpha_{S}^{2}\right)$ (only asymptotic limits [19] have been calculated). These coefficient functions are needed in our GM-VFNS at low $Q^{2}$ at NLO, and at all $Q^{2}$ at NNLO - though in the latter case the definition of the GM-VFNS means that the $\ln \left(Q^{2} / m_{c}^{2}\right)$ terms are subtracted, and the $\mathcal{O}\left(m_{c}^{2} / Q^{2}\right)$ terms die away at high $Q^{2}$, so the GM-VFNS coefficient functions tend to the precisely known massless limits for large $Q^{2} / m_{c}^{2}$.

The initial proposal to deal with this, outlined in [12], was to assume that the mass-dependence in the $\mathcal{O}\left(\alpha_{S}^{2}\right)$ coefficient functions is the same as for the neutral current functions, but with the threshold in $4 m_{c}^{2}$ replaced by a threshold in $m_{c}^{2}$. It was noted that this meant that the coefficient functions at least satisfy the threshold requirements, and tend smoothly to the correct massless limits, so were very likely to be an improvement on the ZM-VFNS. However, in the course of the analyses performed in the latest global fit we have noticed various complications. One consideration is that the neutrino cross sections are given by expressions where $F_{2}\left(x, Q^{2}\right), F_{3}\left(x, Q^{2}\right)$ and $F_{L}\left(x, Q^{2}\right)$ are all important and in the heavyflavour contribution there are significant cancellations between them, so all need to be treated very carefully.

Let us first discuss $F_{2}^{\mathrm{CC}}\left(x, Q^{2}\right)$. At $\mathcal{O}\left(\alpha_{S}^{2}\right)$ this is dominated by the gluon contribution. The simple prescription, suggested in [12], gives large corrections to $F_{2}^{\mathrm{CC}}\left(x, Q^{2}\right)$ at low $Q^{2}$ because the lower threshold compared to the neutral current case leads to a longer convolution length. However, let us consider the comparison of the gluon coefficient functions at $\mathcal{O}\left(\alpha_{S}\right)$, represented by the two diagrams in Fig. 4.

The neutral-current coefficient function is infrared finite and positive. On the other hand the charged-current coefficient function diverges due to the collinear emission of a light (i.e. strange) quark. After subtraction of this divergence, via the usual factorization theorem in $\overline{\mathrm{MS}}$ scheme, there is an approximate factor of $(1+2 \log (1-z))$ in the coefficient function at low $Q^{2}$. It is negative for high $z$ even in the FFNS. At higher $Q^{2}$, the 

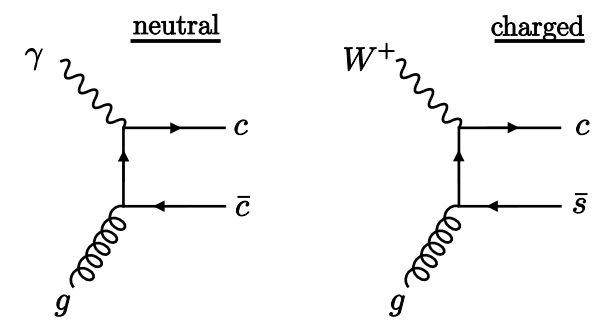

Figure 4. The gluon coefficient function at $\mathcal{O}\left(\alpha_{S}\right)$ in $\mathrm{NC}$ and $\mathrm{CC}$ processes

finite parts of the neutral-current and chargedcurrent GM-VFNS coefficient functions, after the subtraction of the $\log \left(Q^{2} / m_{c}^{2}\right)$ terms, converge to the same massless expression, but from qualitatively different forms at lower $Q^{2}$.

At $\mathcal{O}\left(\alpha_{S}^{2}\right)$, the neutral-current FFNS coefficient function is positive with threshold log enhancements. On the other hand, obtaining the approximate charged-current expression by just a change in threshold limits leads to a large contribution, which, bearing in mind the above discussion, is unlikely to be accurate. At $\mathcal{O}\left(\alpha_{S}^{2}\right)$, the charged-current diagram will still have an extra emission of a strange quark and a corresponding collinear subtraction. So we choose to obtain the charged-current coefficient function by both the change in threshold kinematics and by introducing a $\left(1+2\left(m_{c}^{2} / Q^{2}\right) \log (1-z)\right)$ factor. This still leads to the correct large $Q^{2} / m_{c}^{2}$ limit, but is very likely to be a more accurate representation of the low $Q^{2}$ region. The same procedure is applied to the similar $\mathcal{O}\left(\alpha_{S}^{2}\right)$ singlet-quark contribution.

We now consider $F_{3}^{\mathrm{CC}}\left(x, Q^{2}\right)$ at $\mathcal{O}\left(\alpha_{S}^{2}\right)$. For massless quarks this contribution is zero for initial gluons and singlet quarks, but the coefficient function $C_{3, g}^{\mathrm{CC}, n_{f}}\left(x, m_{c}^{2}, Q^{2}\right)$ is non-zero for finite $Q^{2} / m_{c}^{2}$. However, it must vanish at both $Q^{2} / m_{c}^{2} \rightarrow \infty$ and $W^{2} / m_{c}^{2} \rightarrow \infty$. Hence, our model for $C_{3, g}^{\mathrm{CC}, n_{f}, 2}\left(x, m_{c}^{2}, Q^{2}\right)$ is weighted by a factor $m_{c}^{2} / \hat{W}^{2}=m_{c}^{2} z /\left((1-z) Q^{2}\right)$, as is the singlet-quark contribution. It is important that a suppression of this type is implemented. Other- wise the contribution is potentially anomalously important at low $Q^{2}$ and $x$.

Finally, there is a complication in the ordering for the longitudinal charged-current heavy flavour production. In the massless limit the lowest-order contribution is

$F_{L}^{\mathrm{CC}, c}\left(x, Q^{2}\right)=\alpha_{S}\left(C_{L, g} \otimes g\left(Q^{2}\right)+C_{L, q} \otimes s\left(Q^{2}\right)\right)$.

However, for a massive quark there is a zerothorder contribution

$F_{L}^{\mathrm{CC}, c}\left(x, Q^{2}\right)=\frac{m_{c}^{2}}{m_{c}^{2}+Q^{2}} s\left(\xi, Q^{2}\right)$,

where $\xi=x\left(1+m_{c}^{2} / Q^{2}\right)$. Note that this is unlike the neutral-current case for $F_{L}\left(x, Q^{2}\right)$, where there was also a zeroth-order contribution. Here it is due to a real physical process, i.e. $W^{+}+s \rightarrow$ $c$, rather than one which only makes sense in the limit where the charm quark is most definitely behaving like a massless parton. Hence, for the charged-current case the zero-order contribution must be included. This means that there is a difference in orders below and above the transition point, i.e. FFNS begins at zeroth order whereas the ZM-VFNS begins at first order - opposite to the case for the neutral current $F_{2}\left(x, Q^{2}\right)$. Again a choice in ordering must be made. We choose to obtain the correct limits in both regimes and to maintain continuity. We use (16) to define the LO contribution for $Q^{2}<m_{c}^{2}$, whereas for $Q^{2}>m_{c}^{2}$ the $\mathrm{LO}$ contribution is defined by

$$
\begin{aligned}
& F_{L}^{\mathrm{CC}, c}\left(x, Q^{2}\right)=\frac{m_{c}^{2}}{m_{c}^{2}+Q^{2}} s\left(\xi, Q^{2}\right) \\
& +\left(1-\frac{m_{c}^{2}}{Q^{2}}\right) \alpha_{S}\left(C_{L, g} \otimes g\left(Q^{2}\right)+C_{L, q} \otimes s\left(Q^{2}\right)\right) .
\end{aligned}
$$

At high $Q^{2}$ the first term dies away leading to the normal massless limit. We can easily generalise the prescription to higher orders by including the next term in the $\alpha_{S}$ expansion both sides of the transition point, with the $\left(1-m_{c}^{2} / Q^{2}\right)$ factor always multiplying the highest-order term in the region above the transition point.

In principle, we would also make some use of the $\mathcal{O}\left(\alpha_{S}^{3}\right)$ charged-current coefficient functions in the same way as we do for neutral currents. However, the region where they make a significant impact is overwhelmingly in the small $x$ 
and $Q^{2}$ regime accessed only by HERA neutralcurrent measurements. The amount of modelling required for these terms in charged-current processes is large. Since they are unlikely to have much effect at all, we simply omit them.

\section{Conclusions}

A well defined version of a GM-VFNS is used as as default for heavy flavours in NLO MRST/MSTW global PDF fits, and has been since [8]. The coefficient functions for heavy quarks are now based on those in the $\operatorname{SACOT}(\chi)$ scheme and this leads to a physically sensible and simple definition of a GM-VFNS. However, the ordering is different in the MRST/MSTW and CTEQ approaches for a variety of quantities. Formally this is a higher order effect, but the phenomenological impact is sometimes large.

We now implement a full NNLO GM-VFNS, which has small amount of necessary modelling which only impacts at low $Q^{2}$. This improves the fit to the lowest $x$ and $Q^{2}$ data and has an important impact on the gluon. Pre 2006 NNLO MRST sets used an approximate NNLO heavy flavour treatment, and are now obsolete.

The recent changes in the TR prescription lead to differences in partons of at most $2 \%$ at NLO. This represents an inherent theoretical uncertainty. In principle this decreases with perturbative order, and it will be an interesting project to compare different definitions of the GM-VFNS at NNLO.

MSTW have also implemented a detailed GMVFNS prescription for charged currents. This needs more modelling at low $Q^{2}$ than the neutral current case, but contains the complete set of physics constraints and is guaranteed to tend to correct asymptotic limit for $Q^{2} \gg m_{H}^{2}$.

\section{REFERENCES}

1. A.D. Martin, W.J. Stirling and R.S. Thorne, Phys. Lett. B 636 (2006) 259

[arXiv:hep-ph/0603143].

2. E. Laenen, S. Riemersma, J. Smith and W.L. van Neerven, Nucl. Phys. B 392 (1993) 162 ;
B.W. Harris and J. Smith, Nucl. Phys. B 452 (1995) 109.

3. M. Buza, et al., Eur. Phys. J. C 1 (1998) 301.

4. W.-K. Tung, H.L. Lai, A. Belyaev, J. Pumplin, D. Stump and C.P. Yuan, JHEP 0702 (2007) 053 [arXiv:hep-ph/0611254].

5. A.D. Martin, W.J. Stirling, R.S. Thorne and G. Watt, Phys. Lett. B 652 (2007) 292 [arXiv:0706.0459 [hep-ph]].

6. M. Aivazis, J.C. Collins, F. Olness and W.K. Tung, Phys. Rev. D 50 (1994) 3102.

7. R.S. Thorne and R.G. Roberts, Phys. Lett. B 421 (1998) 303, Phys. Rev. D 57 (1998) 6871.

8. A.D. Martin, R.G. Roberts, W.J. Stirling and R.S. Thorne, Eur. Phys. J. C 4 (1998) 463 [arXiv:hep-ph/9803445].

9. R.S. Thorne and W.-K. Tung, arXiv:0809.0714 [hep-ph].

10. A. Chuvakin, J. Smith and W.L. van Neerven, Phys. Rev. D 61 (2000) 096004.

11. W.-K. Tung et al., J. Phys. G 28 (2002) 983; S. Kretzer et al., Phys. Rev. D 69 (2004) 114005.

12. R.S. Thorne, Phys. Rev. D 73 (2006) 054019 [arXiv:hep-ph/0601245].

13. E. Laenen and S. Moch, Phys. Rev. D 59 (1999) 034027.

14. S. Catani, M. Ciafaloni and F. Hautmann, Nucl. Phys. B 366 (1991) 135.

15. A. Aktas et al. [H1 Collaboration], Eur. Phys. J. C 45 (2006) 23.

16. A.D. Martin, R.G. Roberts, W.J. Stirling and R.S. Thorne, Phys. Lett. B 531 (2002) 216.

17. A.D. Martin, R.G. Roberts, W. J. Stirling and R.S. Thorne, Phys. Lett. B 604 (2004) 61.

18. M. Kramer, F.I. Olness and D.E. Soper, Phys. Rev. D 62 (2000) 096007 [arXiv:hep$\mathrm{ph} / 0003035]$.

19. M. Buza and W.L. van Neerven, Nucl. Phys. B 500 (1997) 301. 